\title{
O bem-estar subjetivo no comportamento de compra de alimentos orgânicos
}

\author{
Subjective Wellbeing in Organic Food Purchase Behavior
}

\author{
Everton Verga \\ Juliano Domingos da Silva ${ }^{\text {II }}$ \\ Solange Alfinito ${ }^{\text {III }}$
}

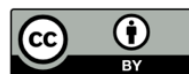

\begin{abstract}
Resumo
Ao longo do século XX pode-se observar mudanças significativas no comportamento dos indivíduos a respeito do consumo de alimentos, e tais alterações apresentam-se em ciclos que demonstraram uma associação entre a alimentação e a qualidade de vida, numa relação direta, abrindo caminho para os alimentos orgânicos. Desse modo a lacuna explorada foi se a perspectiva de bem-estar subjetivo do indivíduo influencia a sua intenção em comprar alimentos orgânicos. Para tanto utilizou-se a Teoria do Comportamento Planejado e a perspectiva do Bem-Estar Subjetivo. O método foi quantitativo, por meio da análise fatorial confirmatória. Os resultados indicaram que o construto Bem-Estar Subjetivo aumentou a explicação da variância da Intenção de Compra, demonstrando sua capacidade preditiva frente a intenção do consumidor.
\end{abstract}

Palavras-chave: Bem-Estar Subjetivo; Teoria do Comportamento Planejado; Intenção de Compra.

\begin{abstract}
Throughout the twentieth century, one can observe consumer behavior changes concerning food consumption, and such changes exhibited in cycles that demonstrate an association between diet and quality of life, in a direct relationship, paving the way for food. Organic. Thus, the gap is explored as an individual's subjective wellbeing perspective that influences their intention to buy organic foods. To do so, use the Theory of Planned Behavior and the Subjective Wellbeing Perspective. The method was quantitative through confirmatory factor analysis. The results indicate that Subjective Welfare increased the explanation of the variation of Purchase Intention, demonstrating its predictive capacity about the consumer's intention.

Keywords: Subjective Wellness; Planned Behavior Theory; Purchase Intent.

' E-mail: evertonmverga@gmail.com, Faculdades Adamantinenses Integradas - FAl, Adamantina/SP [Brasil].

II E-mail: jdomingues8@gmail.com, Universidade Estadual de Maringá, Maringá/PR [Brasil].

III E-mail: solange.alfinito@gmail.com, Universidade de Brasília - PPGA-UNB, Brasília/DF [Brasil].
\end{abstract}

(1) ORCID Id: https://orcid.org/0000-0002-4168-0247

(1) ORCID Id: https://orcid.org/0000-0001-7599-0617

(1) ORCID Id: https://orcid.org/0000-0001-6235-7564

Recebido em: 20/12/2019

Aprovado em: 12/08/2020

\section{Revista Administração em Diálogo}

ISSN 2178-0080

Programa de Estudos Pós-Graduados em Administração

Pontifícia Universidade Católica de São Paulo 


\section{Introdução}

Ao longo do século XX ocorreram mudanças significativas no comportamento dos indivíduos a respeito do consumo de alimentos. Essas mudanças apresentam-se em ciclos que demonstraram uma associação entre a alimentação e qualidade de vida. Nessa seara, as escolhas alimentares indicam ações que refletem na busca por benefícios físicos ou emocionais. No contexto atual, tais assuntos são amplamente debatidos e uma das vertentes desse caminho, de múltiplas rotas, tem se direcionado para a análise do consumo de alimentos. Principalmente nos últimos anos, devido às informações associadas às substâncias tóxicas nos alimentos, consumidores optaram por alimentos sem produtos sintéticos, abrindo caminho para os alimentos de origem orgânica (Hughner et al., 20O7; Thøgersen, 2OII).

Sob essa perspectiva, pesquisas relacionadas ao comportamento dos consumidores a respeito dos produtos orgânicos foram conduzidas em diversos países da União Europeia e nos Estados Unidos (Krystallis \& Chryssohoidis, 2005). Esses estudos, em sua maioria, investigavam como os consumidores percebiam o conceito de orgânicos, bem como as questões referentes à demanda, a atitude dos consumidores frente aos alimentos orgânicos e os fatores que facilitavam ou dificultavam a aceitação desses produtos (Krystallis \& Chryssohoidis, 2005). Outrossim, tais pesquisas revelaram que grande parte dos motivos de compra é atribuída às preocupações ambientais com a saúde, a segurança e a qualidade dos alimentos (Nuttavuthisit \& Thøgersen, 2OI7; Yadav \& Pathak, 20I6; Rana \& Paul, 2OI7).

Diante desse contexto, observa-se que há espaço para se discutir um fator motivador que apresente capacidade de impulsionar os indivíduos a gerar as suas atitudes frente aos alimentos orgânicos. Sendo assim, a inquietação volta-se para as crenças e preocupações com a saúde do consumidor, as quais interferem na sua intenção de compra. Desse modo, a pergunta que se destaca é: a perspectiva de bemestar subjetivo do indivíduo influencia a sua intenção em comprar alimentos orgânicos?

Levando em conta a crescente preocupação dos indivíduos em relação às perspectivas associadas à alimentação saudável, bem como a preocupação com a qualidade de vida e o interesse em compreender os elementos que influenciam as atitudes do indivíduo quando associadas à compra de alimentos orgânicos, os caminhos 
ainda não explorados nas pesquisas realizadas sobre a compra de alimentos orgânicos podem e devem ser problematizados.

É fato inconteste que essa lacuna de investigação se justifica, visto que a explicação sobre a hipótese do Bem-Estar Subjetivo de influenciar o consumo de alimentos orgânicos não foi identificada, mas de certo modo foi evidenciada como uma possibilidade de investigação, haja vista que ainda há um número reduzido de estudos que abordam o tema Bem-Estar Subjetivo na literatura concernente ao consumo de alimentos orgânicos (Apaolaza et al., 20ı8). O espaço que essa pesquisa investiga se sustenta na hipótese de que o Bem-Estar Subjetivo possa representar o antecedente impulsionador do comportamento de compra ou mesmo aquele que aumenta a intenção de comprar alimentos orgânicos. Nesse sentido, o estudo de Apaolaza et al. (20I8) destaca a relação do Bem-Estar Subjetivo como um antecedente, mas, por outro lado, o que se diferencia é a associação desse construto com a intenção de compra, de modo que este possa contribuir com as explicações que ainda permanecem sem justificativas quando se discute a força que influencia a atitude do indivíduo em decidir pela necessidade em comprar e consumir um alimento de origem orgânica (Scalco et al., 2OI7).

Desse modo, com base na afirmativa de Diener et al. (I999), na qual a busca pelo bem-estar é algo constante, realizada pelos indivíduos, e levando em conta que os alimentos podem ser um desses caminhos, como mencionado por Apaolaza et al. (2OI8), o objetivo central desta pesquisa é analisar a intenção de compra de alimentos orgânicos com a inclusão do Bem-Estar Subjetivo como dimensão da Teoria do Comportamento Planejado.

Assim, Ares et al. (20I6) destaca que é possível que o Bem-Estar Subjetivo associado ao contexto dos alimentos exerça influência nas escolhas dos consumidores. Para essa compreensão, identifica-se a relevância da estrutura da Teoria do Comportamento Planejado (TCP), visto que, segundo Scalco et al. (2OI7), essa teoria é uma das mais exploradas nas pesquisas direcionadas à compreensão do comportamento dos consumidores de alimentos orgânicos, sendo uma estrutura viável para mensurar a intenção de compra dos consumidores desse tipo de alimento. Dessa forma, compreender como os consumidores percebem a influência dos alimentos no 
seu Bem-Estar Subjetivo é uma percepção nova no campo de pesquisa (Guillemin et al., 20I6), possibilitando novos modelos estratégicos para incentivar as pessoas a se engajar àqueles hábitos alimentares mais saudáveis como uma das saídas para a melhora da saúde pública (Ares et al., 2015).

\section{Referencial teórico}

Segundo Ajzen (I99I), a TCP é uma extensão da Teoria da Ação Racional (TAR) de Fishbein e Ajzen, de I975, devido às limitações do modelo original que não consideravam os comportamentos sobre os quais as pessoas têm controle (Scalco et al., 20I7). Paul et al. (20I6) ressaltam que a TAR assume a Intenção de Compra como o preditor individual mais importante do comportamento humano, devido à capacidade de lidar com as ações racionais pelo uso sistemático das informações disponíveis (Paul et al., 20I6).

Nesse contexto, a TCP é um modelo psicológico que tem como fator central a intenção do indivíduo de realizar um determinado comportamento. Tal intenção é assumida para capturar os efeitos motivacionais que influenciam o comportamento, e esse comportamento indica o quão as pessoas estão dispostas a tentar e o quanto de esforço elas estão planejando exercer, a fim de executá-lo (Ajzen, ı99i; Paul et al., 20ı6). Considerando essa dinâmica teórica, Ajzen (I99I) ressalta que, como regra geral, quanto mais forte a intenção de envolver-se em um comportamento, maior a probabilidade de que esse comportamento seja realizado (Ajzen \& Fishbein, 2005).

Visto o contexto, é necessário destacar que a TCP leva em consideração três aspectos fundamentais do comportamento humano: Atitude, Normas Subjetivas e Controle Comportamental Percebido (Ajzen, r99i; Paul et al., 20I6; Scalco et al., 20I7). Esses são os antecedentes básicos da intenção de se engajar em um determinado comportamento real (Scalco et al., 2OI7).

Por fim, na visão de Yadav e Pathak (20I6), a TCP é considerada uma das estruturas mais úteis para explicar o comportamento humano, pois os indivíduos são guiados por crenças comportamentais, crenças normativas e de controle, que resultam em ações como a Atitude em relação ao Comportamento de Compra, as Normas Subjetivas e o Controle Percebido. Desse modo, a seguir será destacado cada um dos 
componentes da TCP presentes na Figura I. Essa descrição é necessária para que se possa compreender como os construtos do modelo se relacionam entre si e o papel deles frente à intenção.

\section{Atitude}

Em consideração aos estudos de Festinger e Carlsmith (I959) sobre a abordagem da teoria da dissonância cognitiva de Festinger, de I957, dimensionam-se contribuições relevantes no que tange à compreensão do mecanismo relacionado à concepção da atitude do indivíduo. Isso porque, segundo os autores, as atitudes são resultado das crenças, e a racionalização das atitudes envolve um processo complexo, devido sobretudo à possibilidade de alterações das crenças do indivíduo e atitudes básicas consolidadas.

Para Ajzen e Fishbein (I977), o comportamento é determinado pela intenção, a qual, por sua vez, é uma função da atitude. Dessa forma, a atitude de um indivíduo é a sua avaliação a respeito de um elemento e influência, por fim, o padrão geral de suas ações, que são executadas considerando um determinado alvo, em um dado contexto e em um ponto no tempo. Esse componente reflete as preferências individuais para executar ou não um comportamento, expressa a avaliação global (positiva/negativa) dos indivíduos sobre determinado comportamento em questão: quanto mais positiva a atitude, mais forte será a intenção de expressar tal comportamento (Scalco et al., 2OI7). A atitude é a emoção psicológica lastreada pelas avaliações dos consumidores, incluindo considerações afetivas (por exemplo, eu gosto, é atraente, etc.) e avaliativas (por exemplo, tem vantagens) (Paul et al., 2OI6).

\section{Normas Subjetivas}

O segundo componente da intenção comportamental é a norma subjetiva. Esse termo é definido como a pressão social percebida para realizar ou não o comportamento (Ajzen, r99i; Paul et al., 20I6). Esse construto capta o sentimento do indivíduo sobre a pressão social que sente em determinado comportamento. Assim, consumidores que apresentam Normas Subjetivas positivas em relação a determinados comportamentos estão mais propensos às intenções positivas (Paul et al., 20I6).

\section{Revista Administração em Diálogo}


Para Scalco et al. (20I7), as normas sociais comuns são percebidas pelos indivíduos em relação a engajar (ou não) um comportamento específico, uma vez que a adesão às normas é importante por permitir que os membros do grupo evitem desencadear respostas de rejeição enquanto estimulam um senso de aprovação social (Scalco et al., 2OI7). Nesse sentido, Teng e Wang (2OI5) ressaltam que, à medida que as tendências da sociedade avançaram para uma alimentação mais saudável, ampliou-se a responsabilidade individual pelo bem-estar pessoal, exercendo, portanto, influência sobre as decisões dos consumidores em comprar alimentos orgânicos. Isso porque, conforme Yadav e Pathak (2OI6), os consumidores consideram as crenças normativas, as quais estão associadas a uma percepção individual sobre como indivíduos mais próximos se comportariam em uma determinada situação, considerando a motivação (juízo positivo/negativo; avaliação de resultados) para cumprir determinadas normas sociais (Ajzen, 199I).

\section{Controle Comportamental Percebido}

Para Paul et al. (20I6), o controle comportamental percebido, que neste estudo será tratado simplesmente como Controle Percebido, refere-se à facilidade ou dificuldade percebida de realizar um comportamento (Ajzen, I99I), refletindo experiências passadas e obstáculos antecipados. Essa variável é associada à capacidade percebida de realizar determinado comportamento e baseia-se em crenças sobre fatores que podem facilitar ou impedir seu desempenho (crenças de controle) (Scalco et al., 2OI7).

Segundo Yadav e Pathak (20I6), o Controle Percebido é um resultado das crenças de controle e do poder percebido. As crenças de controle são entendidas como a crença do indivíduo em relação à presença de certos fatores que facilitam ou impedem o desempenho de um comportamento específico (por exemplo, tempo, dinheiro, oportunidade). O poder percebido, por sua vez, refere-se à avaliação pessoal do impacto desses fatores ou impedimento no comportamento particular (Ajzen, I99I).

Por fim, Scalco et al. (20I7) indicam que estudos se concentram em medir o Controle Percebido por meio da disposição pessoal e da facilidade de compra de alimentos orgânicos, em vez de investigarem a influência de barreiras como preços 
mais elevados e menor disponibilidade, por exemplo. Assim, considerando o contexto, há pontos a serem explorados com relação ao controle comportamental percebido na intenção de compra associada à alimentos orgânicos.

\section{Intenção Comportamental}

Segundo Yadav e Pathak (20I6), a intenção comportamental é uma indicação da prontidão do indivíduo para realizar um determinado comportamento, supondo-se que esse elemento seja um antecedente imediato do comportamento. Com isso, quanto mais o indivíduo for propenso à atitude diante de um comportamento, mais favorável à norma subjetiva será, e maior o controle comportamental percebido, enfim. Assim, mais intensa será a intenção em se efetivar o comportamento.

Para Taufique e Vaithianathan (2OI8), a intenção comportamental refere-se ao grau de determinação e disposição de um indivíduo para realizar um comportamento específico. A intenção comportamental é frequentemente associada às Atitudes e Normas Subjetivas. Ou seja, quanto maior os níveis de Atitude e Normas Subjetivas, maiores são as chances de um indivíduo realizar o comportamento. Portanto, é necessário destacar que a Atitude, a Norma Subjetiva e o Controle Percebido desempenham um papel significativo na determinação da Intenção de Compra dos consumidores (Yadav \& Pathak, 20I6).

Assim, considerando a relevância do modelo da TCP no contexto da mensuração da intenção e a possibilidade de influência dos alimentos orgânicos na percepção do Bem-Estar Subjetivo (Apaolaza et al., 20I8), há de se discutir se o bemestar tem relação, em algum nível, com a intenção desses consumidores. Desse modo, é preciso apresentar a contextualização do construto Bem-Estar Subjetivo.

\section{Bem-Estar Subjetivo}

Existe uma lacuna na discussão temática sobre a questão do consumo de orgânicos em relação à melhoria do Bem-Estar Subjetivo (Apaolaza et al., 20I8). Portanto, é necessário considerar as discussões e os pontos de vista teóricos sobre o bem-estar, suas dimensões Físico Social, Emocional, Intelectual e Espiritual, e o seu papel no contexto dos alimentos. 
O Bem-Estar Subjetivo não é uma entidade unitária. Ela apresenta múltiplas facetas que devem ser avaliadas por meio de julgamentos globais, relatos momentâneos de humor, fisiologia, memória e expressões emocionais (Diener et al., I999). Nesse sentido, Diener et al. (I999) destacam que a satisfação é o elo central do bem-estar e que, para alcançá-la, é necessário um nível de aspirações influenciadas pelas experiências passadas, comparações com os outros, valores pessoais, dentre outros fatores.

Para melhor analisar o Bem-Estar Subjetivo, Diener et al. (1985) definem três componentes centrais: o afeto positivo, o afeto negativo e um componente cognitivo, referindo-se à satisfação com a vida. O primeiro e o segundo componentes avaliam os aspectos emocionais do bem-estar (o humor agradável e desagradável) e são mais flutuantes em comparação com o último componente, o qual é entendido como uma avaliação global da qualidade de vida de uma pessoa de acordo com seus critérios escolhidos (Diener et al., I985).

Segundo Guillemin et al. (20I6), não existe uma definição padrão para bemestar. Entretanto, há um consenso que atravessa as múltiplas definições disponíveis: todas elas concordam a respeito de que o bem-estar é um conceito multidimensional e complexo, associado aos estágios psicológicos e somáticos que envolvem aspectos físicos, emocionais, sociais, espirituais e intelectuais (Ares et al., 20I5; Guillemin et al., 20I6). Do ponto de vista teórico, o bem-estar representa o que é bom para um indivíduo e, por isso, tem-se argumentado que as nossas escolhas e decisões de estilo de vida estão orientadas para a obtenção do bem-estar (Ares et al., 20I6).

\section{Dimensões do Bem-Estar}

Ares et al. (20I6) observam que há um consenso sobre o fato de que o bemestar é o resultado de um estado somático, o qual envolve fatores relacionados à saúde física, estados emocionais, apoio social, espiritual e estímulos intelectuais. Greenberg (I985) destacava essas cinco dimensões mencionadas por Ares et al. (2OI6), ao afirmar que o bem-estar é a integração dos cinco componentes, que convergem, efetivamente, em um único ponto central associado ao contínuo de saúde-doença ligado à condição de bem-estar do indivíduo. 
Para Greenberg (1985), o bem-estar é um construto que se alcança por meio do equilíbrio na integração das cinco dimensões. No entanto, o autor destaca que há assimetrias. Ele enfatiza que esse foco apenas na dimensão física ocasiona uma desigualdade no equilíbrio do bem-estar ao ponto que a saúde física se infla frente às outras dimensões, afetando o equilíbrio. Assim, do mesmo modo com que os indivíduos trabalham para melhorar a sua saúde física, eles também precisam trabalhar para melhorar as outras dimensões do bem-estar.

Greenberg (1985) observa, desse modo, que há uma tendência voltada para a saúde física do indivíduo, porém, ao direcionar o foco apenas em uma dimensão, há possibilidade de se perder o equilíbrio que sustenta o bem-estar. Ao manter esforços concentrados somente na questão física, sem a integração com as demais dimensões, coloca-se em risco a dinâmica da integração para o alcance do bem-estar. Por exemplo, é possível praticar exercícios físicos com amigos e cônjuge para melhorar a saúde social e emocional, ler livros sobre aptidão física para melhorar a saúde mental, exercitar-se em parques e caminhos da natureza para experimentar a ordem do universo e melhorar a saúde espiritual. Por fim, a lição é que, ao se melhorar um componente, é necessário, conscientemente, trabalhar para melhorar todos os outros, cultivando a simetria das condições de bem-estar do indivíduo.

\section{Procedimentos metodológicos}

Essa pesquisa caracteriza-se do tipo quantitativa, descritivas e realizada por meio de uma survey. A população estabelecida é de consumidores brasileiros de diferentes idades (acima de I8 anos), sexo, classes econômicas e regiões do país, os quais se declarassem compradores de alimentos de origem orgânica. Para tal, aplicouse o critério utilizado por Marôco (2OIO), segundo o qual, para se garantir a variabilidade suficiente de estimação dos parâmetros do modelo, em um modelo de equações estruturais, deve-se ter entre Io a I5 observações por cada variável manifesta. Desse modo, considerando os instrumentos usados, a serem detalhados mais à frente, o número de participantes total foi de $\mathrm{N}=639$ válidos. Destaca-se que o processo de amostragem foi não probabilístico, por conveniência. Aplicados a consumidores de todas as regiões do país, divididos entre 325 (5 I\%) do sexo feminino e 3 I4 (49\%) do 
sexo masculino, com níveis de escolaridade do ensino fundamental incompleto a pósgraduação, com renda de um salário mínimo até vinte salários, apresentando uma média de idade de 3I,55 anos com desvio padrão de IO,99 anos.

Instrumento: A aplicação do questionário iniciou com uma pergunta filtro: “Você já comprou ou compra algum tipo de alimento orgânico?". Posteriormente foram as questões das escalas e, por fim, os elementos sociodemográficos. A primeira escala do instrumento foi de Al-Swidi et al. (20I4), que abordou a TCP. A segunda escala tratou o Bem-Estar Subjetivo para o contexto dos alimentos e foi desenvolvida por Ares et al. (20I6). Vale ressaltar que, para ambos os questionários, a escala utilizada foi do tipo Likert com sete pontos (de I - Discordo totalmente a 7 - Concordo totalmente) para captar a opinião dos participantes em relação aos itens do instrumento. A escala da TCP de Al-Swidi et al. (2OI4) é originalmente composta por 4 construtos, totalizando 22 itens: Atitude (8 perguntas), Comportamento Percebido (6 perguntas), Normas Subjetivas (4 perguntas) e Intenção de Compra (4 perguntas). Essa escala foi escolhida devido à sua abordagem direcionada para a Intenção de Compra de alimentos orgânicos, visto que o estudo de Al-Swidi et al. (20I4) emprega o modelo original da TCP proposto por Ajzen (I99I).

Em relação à escala de mensuração do Bem-Estar Subjetivo de Ares et al. (20I6), ela é composta por seis dimensões: o bem-estar Geral (2 itens), o bem-estar Físico (7 itens), o bem-estar Intelectual (5 itens), o bem-estar Emocional (7 itens), o bem-estar Social (5 itens) e o bem-estar Espiritual (5 itens), totalizando 3 I itens da escala. Desse modo, a escolha da escala de Ares et al. (20I6) levou em conta a sua referência a partir de outros trabalhos que exploram o tema do Bem-Estar Subjetivo, como Meiselman (20I6), por citar a escala como um meio eficiente de mensuração do bem-estar no contexto alimentar

Procedimentos de Coleta de Dados e Análise: A coleta de dados foi realizada de forma eletrônica, com o uso da plataforma HeapUp, utilizando-se um questionário autoaplicável, por meio de link de acesso, o qual poderia ser respondido via desktop ou dispositivo mobile. A pesquisa teve início no dia 7 de janeiro de 2019 e foi concluída em 24 de janeiro de 2019 .

\section{Revista Administração em Diálogo}


Quanto a análise considerou-se o critério do Listwise, e a avaliação descritiva dos dados, visando observar a normalidade (coeficientes de assimetria e curtose) e a validade (convergente e discriminante) dos dados coletados e considerando as estatísticas de média e desvio padrão, cujos valores estão descritos na seção dos resultados. As análises dos dados foram feitas por meio da aplicação de métodos estatísticos multivariados, a partir de análise fatorial e teste analítico de modelagem de equações estruturais (Structural Equation Modeling - SEM), com base em Covariâncias (CB-SEM) e seguindo o protocolo de Hair et al. (2OI4), com a utilização dos softwares IBM SPSS Statitics $20{ }^{\circledR}$ e IBM SPSS Amos $20 \AA$. Os dados obtidos foram tabulados em planilha eletrônica do software Microsoft Office Excel $2013{ }^{\circledR}$, dirigido ao exame de possíveis valores ausentes.

Com o intuito de atestar a validade e a confiabilidade das escalas de Al-Swidi et al. (2OI4) e Ares et al. (2OI6), foram utilizados os procedimentos de análise dos itens e dos construtos por meio dos testes do Alfa de Cronbach (Hair et al., 2009), Rho de Jöreskog (Chin, I998) e a Confiabilidade Composta (Marôco, 2OIO).

\section{Resultados}

A observação dos dados seguiu o rigor estatístico, sob a visão dos requisitos estabelecidos para aplicação de análises multivariadas (Marôco, 20IO). Esta contou inicialmente com 729 respondentes. Foram excluídos 90 casos de dados omissos que foram excluídos, totalizando, portanto, uma amostra final de 639 respondentes, número esse que atende aos pressupostos de Marôco (2OIO). Quanto aos outliers, não foram identificados outliers univariados.

Levando em conta a hipótese nula de que os dados se originam de uma distribuição normal, a análise de todas as variáveis que compõem as escalas da TCP e Bem-Estar Subjetivo mostrou que a curtose mínima foi de -I,83 e a máxima de -0,062 e a curtose foi com valor mínimo de -I,33 e máxima de 2,55. Tais resultados indicam que se pode pressupor a normalidade dos dados, conforme Marôco (20IO).

AFC da Teoria do Comportamento Planejado. O modelo inicial da TCP apresentou os seguintes resultados: $\chi^{2}=806,24 ; \chi^{2} /$ g.l. $=4,243 ; \mathrm{CFI}=0,879 ; \mathrm{TLI}=$ o,9I2; RMSEA=O,O7I; p-valor ${ }^{2}=$ o,ooo. Para um ajuste adequado, Hair et al. (2009) 
destacam que as estimativas de cargas padronizadas devem ser acima de $(\lambda<0,70)$.

Desse modo, excluiu-se dois itens da dimensão de Atitude, dois itens da dimensão

Controle Percebido e um item da dimensão Normas Subjetivas. Seguindo a validação

fatorial do modelo da TCP, foram analisadas a validade convergente e discriminante

com base no cálculo da variância extraída média (VEM) e a confiabilidade composta

(CC). Os resultados são apresentados na Tabela I.

Tabela I. Validade convergente e discriminante da escala TCP

\begin{tabular}{lcccc}
\hline Variáveis & $\begin{array}{c}\text { Intenção de } \\
\text { Compra }\end{array}$ & Atitude & $\begin{array}{c}\text { Controle } \\
\text { percebido }\end{array}$ & $\begin{array}{c}\text { Norma } \\
\text { subjetiva }\end{array}$ \\
\hline Validade Convergente & & & & \\
Variância Extraída Média (VEM) & 0,70 & 0,58 & 0,60 & 0,64 \\
Confiabilidade Composta (CC) & 0,90 & 0,89 & 0,82 & 0,84 \\
Alfa de Cronbach & 0,90 & 0,90 & 0,82 & 0,84 \\
Rho de Joreskog & 0,90 & 0,89 & 0,82 & 0,84 \\
Validade Discriminante - Critério de Fornell-Larcker* & & & \\
1. Intenção & 0,84 & & & \\
2. Atitude & 0,82 & 0,76 & & \\
3. Norma subjetiva & 0,62 & 0,54 & 0,77 & \\
4. Controle percebido & 0,64 & 0,58 & 0,63 & 0,80 \\
\hline
\end{tabular}

Nota. *Números na diagonal principal são as raízes quadradas das variâncias extraídas médias (VEM) e os números fora da diagonal são os valores das correlações entre as dimensões do construto. Os índices dos Alfas de Cronbach foram calculados após a AFC.

Inicialmente, os dados demonstram que há confiabilidade composta para todos os construtos da escala da TCP, uma vez que os resultados foram superiores a o,8o, atingindo valores acima de o,70 (Marôco, 2OIO), e, de forma complementar, foram recalculados os Alfas de Cronbach e Rho de Joreskog, todos superiores ao limite inferior de 0,7O mencionado por Hair et al. (2009) e Chin (1998). Conclui-se, portanto, que os construtos da escala são confiáveis e válidos no modelo da TCP e para a aplicação no modelo geral da pesquisa, com índices de ajustes $\chi^{2} /$ g.l. = 2,248; CFI = o,982; TLI $=, 976 ;$ RMSEA $^{=} \mathrm{O}, \mathrm{O} 44 ; \mathrm{p}^{- \text {valor }^{2}}=\mathrm{o}, \mathrm{ooo}$.

AFC do Bem-Estar Subjetivo. O modelo inicial apresentou os seguintes resultados significativos: $\chi^{2} /$ g.l. $=$ IO,330; CFI = o,8I8; GFI = o,6I9; TLI = o,798; RMSEA $=$ O,I2I; p-valor ${ }^{2}=0,000$, os quais demonstram certas inconsistências (Marôco, 20Io). Para obter ajustes adequados, foram excluídos dois itens da dimensão Emocional, um item da dimensão Social, um item da dimensão Física e dois itens da 
dimensão Intelectual. Após as rodadas de análise o modelo final apresentou os seguintes resultados: $\chi^{2}$ /g.l. = 3,530; CFI = o,968; GFI = o,9I3; TLI = o,96o; RMSEA = o,o63; p-valor $^{2}=$ o,ooo. Essas informações confirmam, assim, a consistência do modelo especificado e indicam que esse modelo se ajusta à população de modo aceitável (RMSEA = o,o62), ou seja, apresenta um bom ajustamento. As análises da validação fatorial do Bem-Estar Subjetivo foram realizadas por meio de análises da validade convergente e discriminante, cujos resultados são apresentados na Tabela 2.

Tabela 2. Validade convergente e discriminante da escala de Bem-Estar Subjetivo

\begin{tabular}{|c|c|c|c|c|c|c|}
\hline Variáveis & $\begin{array}{l}\text { Bem-Estar } \\
\text { Intelectual }\end{array}$ & $\begin{array}{l}\text { Bem-Estar } \\
\text { Físico }\end{array}$ & $\begin{array}{l}\text { Bem-Estar } \\
\text { Emocional }\end{array}$ & $\begin{array}{c}\text { Bem-Estar } \\
\text { Social }\end{array}$ & $\begin{array}{l}\text { Bem-Estar } \\
\text { Espiritual }\end{array}$ & $\begin{array}{r}\text { Bem-Est } \\
\text { Geral }\end{array}$ \\
\hline \multicolumn{7}{|l|}{ Validade Convergente } \\
\hline Variância Extraída Média (VEM) & 0,84 & 0,72 & 0,78 & 0,72 & 0,80 & 0,70 \\
\hline Confiabilidade Composta (CC) & 0,94 & 0,94 & 0,91 & 0,89 & 0,89 & 0,92 \\
\hline Alfa de Cronbach & 0,94 & 0,94 & 0,91 & 0,88 & 0,88 & 0,92 \\
\hline Rho de Joreskog & 0,94 & 0,93 & 0,91 & 0,88 & 0,92 & 0,88 \\
\hline \multicolumn{7}{|c|}{ Validade Discriminante - Critério de Fornell-Larcker* } \\
\hline 1. Bem-Estar Intelectual & 0,92 & & & & & \\
\hline 2. Bem-Estar Físico & 0,91 & 0,85 & & & & \\
\hline 3. Bem-Estar Emocional & 0,83 & 0,83 & 0,88 & & & \\
\hline 4. Bem-Estar Social & 0,50 & 0,40 & 0,63 & 0,85 & & \\
\hline 5. Bem-Estar Espiritual & 0,72 & 0,67 & 0,87 & 0,82 & 0,90 & \\
\hline 6. Bem-Estar Geral & 0,76 & 0,89 & 0,80 & 0,33 & 0,64 & 0,84 \\
\hline
\end{tabular}

Nota. * Números na diagonal principal são as raízes quadradas das variâncias extraídas médias (VEM) e os números fora da diagonal são os valores das correlações entre as dimensões do construto. Os índices dos Alfas de Cronbach foram calculados após a AFC.

Com os dados apresentados na Tabela 2, conclui-se que todas as dimensões atingiram índices aceitáveis de confiabilidade composta, pois os resultados foram superiores a o,80, cujo índice é superior ao mínimo aceitável por Marôco (2OIO). Os Alfas de Cronbach foram recalculados com os itens resultantes da AFC para todas as dimensões, todos com valores superiores ao limite inferior de o,70 mencionado por Hair et al. (2009) e Rho de Jöreskog superiores a o,7o (Chin, I998). No processo de avaliação da validade discriminante, foi observado que apenas a dimensão Bem-Estar Geral não se diferenciou, considerando os critérios de Marôco (2OIO), ou seja, a variância compartilhada do Bem-Estar Geral foi superior à VEM do Bem-Estar Físico, visto que ambas foram altamente correlacionadas. Entretanto, considerando os outros 
resultados, como a confiabilidade composta e a validade convergente, pode-se concluir que o resultado desfavorável no requisito da validade discriminante não invalida a dimensão do Bem-Estar Geral.

Modelo Geral da TCP e Bem-Estar Subjetivo. Após os procedimentos de análises confirmatórias das escalas da TCP e do Bem-Estar Subjetivo, o passo seguinte foi a avaliação do Modelo Geral, o qual, por sua vez, visa responder aos objetivos e às hipóteses apresentadas neste trabalho. Desse modo, o primeiro teste do modelo proposto, com os seguintes resultados: $\chi^{2}=\left(65^{2}\right) 2692,75 \mathrm{I} ; \chi^{2} /$ g.l. $=4, \mathrm{I} 3 \mathrm{O} ; \mathrm{CFI}=$ o,9o6; GFI = o,770; TLI = o,899; RMSEA = o,o7o; p-valor ${ }^{2}=0,000$, indica um resultado dentro das fronteiras aceitáveis quando ajustado, considerando os critérios de avaliação. No entanto, ainda se encontra discretamente fora dos padrões descritos por Marôco (2OIO), uma vez que a busca é por modelos explicativos com bons ajustes globais. Seguindo o procedimento de análise, foram considerados os índices de modificação (superiores a II, $\mathrm{p}<$ o, OOI) do AMOS e, nesse caso, observou-se que os ajustes foram concentrados nas dimensões do Bem-Estar Subjetivo.

Na Figura I, nota-se que os ajustes se concentraram no construto Bem-Estar Subjetivo. Isso ocorre devido à existência de um construto de segunda ordem latente. No Bem-Estar Subjetivo, de acordo com Marôco (2OIO), podem ocorrer correlações consideráveis entre fatores de primeira ordem e correlações de erros que saturam em fatores diferentes. Sendo assim, na escala do Bem-Estar Subjetivo, é plausível a defesa teórica de questões que possam surgir quanto à correlação entre os erros das dimensões. Frente a isso, os resultados do modelo global reespecificado $\chi^{2} / g .1$. = 2,707; CFI = o,95o; GFI = o,86o; TLI = o,950; RMSEA = o,o5o; p-valor ${ }^{2}=0,000$, indicam que, nos índices gerais, o modelo final apresenta valores acima dos critérios estabelecidos pela literatura, ou seja, a qualidade de ajustamento global do modelo fatorial representa, de fato, o objetivo proposto, visto que esses resultados corroboram a inclusão do construto Bem-Estar Subjetivo como viável dentro do modelo da TCP. Isso porque o modelo global apresentou o ajuste necessário, indicando que a sua estrutura final é passível de análise e replicação.

Após a validação das escalas, apresenta-se os resultados do modelo global do modelo de equação estrutural baseado em covariância (CB-SEM). A Figura I, além de 
destacar as cargas fatoriais, indica os coeficientes de regressão entre os construtos e os respectivos $\mathrm{R}_{2}$ do modelo.

Figura I. Modelo global reespecificado

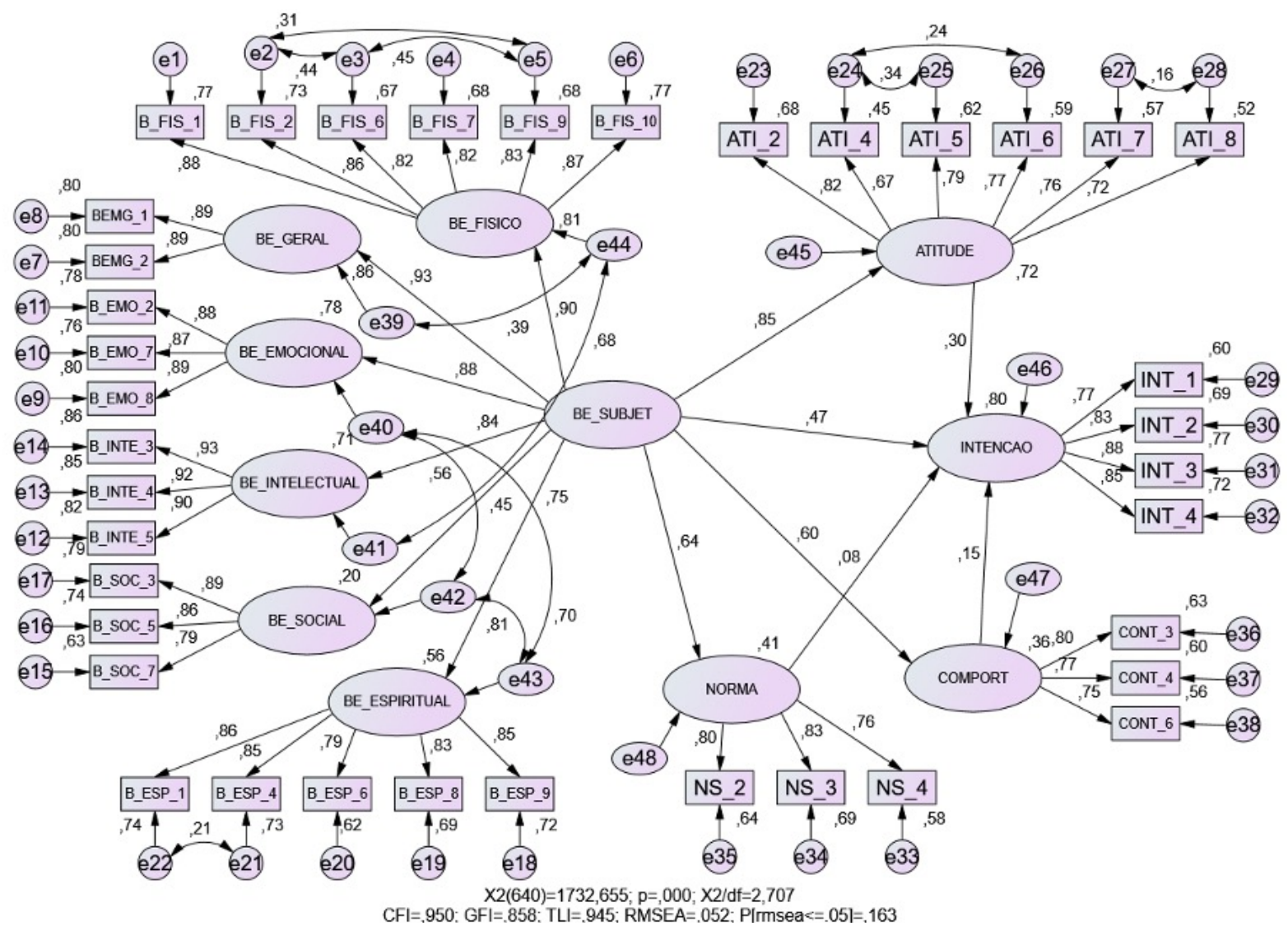

Na Tabela 3 são apresentadas as estimativas das relações entre o Bem-Estar Subjetivo e a TCP, apontando, na coluna do p-valor, que todas as relações foram significativas para um nível de $5 \%$. 
Tabela 3. Estimativas do modelo global

\begin{tabular}{|c|c|c|c|c|c|c|c|}
\hline & & & Estimativa & $\bar{B}$ & $\begin{array}{l}\text { S.E. } \\
\text { S. }\end{array}$ & C.R. & $\overline{p \text {-valor }}$ \\
\hline Bem-Estar Subjetivo & $\rightarrow$ & Normas Subjetivas & 0,719 & 0,639 & 0,054 & 13,391 & 0,001 \\
\hline Bem-Estar Subjetivo & $\rightarrow$ & Atitude & 0,824 & 0,847 & 0,047 & 18,078 & 0,001 \\
\hline Bem-Estar Subjetivo & $\rightarrow$ & Controle Percebido & 0,740 & 0,603 & 0,057 & 12,907 & 0,001 \\
\hline Bem-Estar Subjetivo & $\rightarrow$ & Intenção de Compra & 0,527 & 0,472 & 0,080 & 6,560 & 0,001 \\
\hline Normas Subjetivas & $\rightarrow$ & Intenção de Compra & 0,080 & 0,081 & 0,037 & 2,135 & 0,033 \\
\hline Atitude & $\rightarrow$ & Intenção de Compra & 0,338 & 0,301 & 0,069 & 4,870 & 0,001 \\
\hline Controle Percebido & $\rightarrow$ & Intenção de Compra & 0,134 & 0,148 & 0,034 & 4,003 & 0,001 \\
\hline
\end{tabular}

Observando os resultados, é possível concluir que o Bem-Estar Subjetivo foi o construto que mais influenciou a Intenção de Compra $(\beta=0,47)$, ou seja, esse fator tem a capacidade de explicar 0,47 da variabilidade da Intenção de Compra. O segundo construto que mais explica a variabilidade da Intenção é a Atitude $(\beta=0,30)$, indicando que, ao se comparar os resultados do modelo canônico da TCP $(\beta=0,62)$ com os valores do modelo global, constata-se uma queda no poder de explicação do construto frente à Intenção. Esse fato é justificável caso se considerem as alegações de Scalco et al. (20I7) de que na TCP a Atitude é o fator que mais exerce influência na intenção, porém, ainda existem incertezas quanto a essa relação. No entanto, a respeito de o Bem-Estar Subjetivo apresentar um alto grau de influência sobre a Atitude $(\beta=0,85)$, pode-se inferir que essas incertezas sejam reduzidas e, com isso, o resultado de $\beta=$ o,3o da Atitude na intenção pode ser explicado pela influência do Bem-Estar Subjetivo como um antecedente da Atitude quanto ao indivíduo expressar a sua avaliação global positiva ou negativa sobre ter uma Atitude de comprar ou não o alimento orgânico.

Os resultados do Controle Percebido $(\beta=\mathrm{O}, \mathrm{I5})$ indicam, por sua vez, uma proximidade quando comparados ao valor do modelo original da TCP testado $(\beta=0,16)$. Ou seja, quando não há a interferência de elementos externos, o construto Controle Percebido contribui com a explicação da variância da intenção, isto é, sem interferências inoportunas, a percepção do indivíduo fomenta a expressão de um comportamento.

Por fim, o último item do modelo global discutido foi o construto Normas Subjetivas, que apresentou o menor grau de influência no modelo global $(\beta=0,08)$, enquanto que, no modelo da TCP, os resultados foram de um $\beta=0,19$, indicando, 
assim, que a inserção do construto Bem-Estar Subjetivo, o qual visa às questões da perspectiva individual, contribuiu para uma redução da influência social frente às decisões dos indivíduos $(\beta=0,63)$, como visto no modelo global. Isso significa que, apesar de o contexto das relações sociais se destacar no processo de decisão que envolve as Normas Subjetivas, essa força não é mais o centro dessa relação, ou seja, o indivíduo considera incialmente a sua vontade, o seu ponto vista sobre o consumo de alimentos orgânicos, e depois observa o que os demais pensam sobre sua Atitude. Esse é um indicativo de que as decisões individuais prevalecem quando considerado o modelo global proposto.

Portanto, nesta pesquisa, constatou-se que o construto Bem-Estar Subjetivo, além de aumentar a explicação da variância da Intenção de Compra $R^{2}$ o,8o, avança para a possibilidade de o modelo esclarecer pontos que ainda permaneciam incertos, como a força que motiva as Atitudes, a qual, nesta pesquisa, foi identificada pela preocupação do indivíduo com o seu próprio bem-estar. A seguir, no próximo capítulo, serão discutidos os testes de Anova, direcionados a identificar as diferenças entres os participantes da pesquisa.

\section{Discussão dos Resultados}

No que se refere aos resultados obtidos, estes apontam que o Bem-Estar Subjetivo influencia positivamente os fatores Atitude, Normas Subjetivas, Controle Percebido e Intenção de Compra, indicando, assim, que o Bem-Estar Subjetivo apresenta uma relação positiva com os construtos que compõem a TCP. Isso significa que o construto do Bem-Estar Subjetivo demonstra a sua associação no modelo da TCP. Essa interação do construto do Bem-Estar Subjetivo com a TCP é justificada por Ajzen (I99I) ao relatar que Atitude, Normas Subjetivas e Controle Percebido são um conjunto apropriado para se medir a intenção, mas a natureza exata dessas relações que antecedem essas variáveis apresenta uma condição de incertezas quanto aos seus motivadores.

Nesse sentido, a significância estatística do Bem-Estar Subjetivo dentro da TCP vai ao encontro da proposta que esta pesquisa sustenta, ou seja, que o Bem-Estar Subjetivo impacta, de forma positiva, a Intenção de Compra de alimentos orgânicos. 
Ajzen (199I) ressalta que a TCP está aberta para modificações que aprofundem e ampliem a capacidade preditiva do modelo, questão essa verificada a partir dos resultados até aqui descritos. Desse modo, Yadav e Pathak (20I6) relatam que, embora a TCP seja baseada na suposição de que a intenção é determinada pela Atitude, Normas Subjetivas e Controle Percebido, essa pesquisa mostra que o Bem-Estar Subjetivo é uma nova variável preditora que melhora a utilidade preditiva da TCP.

Além disso, analisando os resultados do Bem-Estar Subjetivo com os construtos da TCP, verificou-se que a Atitude foi o fator mais influenciado pelo bemestar, e possivelmente essa força está ligada ao fato destacado por Arvola et al. (2008) de o modelo da TCP ser insuficiente em considerar as influências afetivas e morais. Sendo assim, Arvola et al. (2008) destacam que os componentes afetivos se referem a sentimentos ou emoções que os indivíduos têm em relação ao objeto de atitude, enquanto o componente cognitivo diz respeito aos pensamentos que esses indivíduos possuem sobre o objeto de atitude.

Dessa forma, o construto Bem-Estar Subjetivo atende a essas duas demandas, pois aborda tanto questões emocionais como os elementos cognitivos guiados por reflexões sobre os possíveis benefícios que, por exemplo, os alimentos orgânicos trazem para a vida do consumidor. Assim, é possível identificar que o Bem-Estar Subjetivo demonstra essa relação forte com as atitudes dos consumidores que se voltam para ações as quais atentem às suas necessidades tanto emocionais quanto racionais de modo que representem suas preferências.

A respeito da influência do Bem-Estar Subjetivo quanto às normas sociais, Arvola et al. (2008) destacam que as normas são vistas como regras socialmente compartilhadas do que é certo e errado. Sendo assim, é possível inferir que o Bem-Estar Subjetivo influencia as normas sociais a ponto de deslocar o eixo descrito por Arvola et al. (2008), de maneira que as questões individuais prevaleçam sob as grupais. Esse argumento é sustentado principalmente pela descrição de Diener et al. (2003) ao ressaltarem que o Bem-Estar Subjetivo se refere a uma avaliação de como os indivíduos percebem suas próprias vidas. Quanto ao Bem-Estar Subjetivo e ao Controle Percebido, Ajzen (I99I) expõe este como o construto que representa diretamente o resultado do sistema de crenças de um indivíduo sobre o poder em um dado contexto e sobre fatos 
internos que influenciam o seu comportamento. Desse modo, considerando que a perspectiva do Bem-Estar Subjetivo trata de um posicionamento individual, associado, em certa medida, à questões cognitivas, é possível justificar essa influência, uma vez que tanto o Controle Percebido quanto o Bem-Estar Subjetivo apresentam o autocontrole como um guia em restringir ou executar um comportamento específico e ambos podem sofrer com barreiras (o preço dos alimentos orgânicos) que influenciam na intenção de comprar.

Por fim, na relação entre o Bem-Estar Subjetivo e a Intenção de Compra, é destacada por Ajzen (I99I) que a intenção é um preditor significativo do comportamento de compra, sendo assim, essa prontidão do indivíduo para realizar determinado comportamento é assumida como um antecedente imediato do comportamento real. Dessa forma, os resultados do modelo geral demonstram que o construto Bem-Estar Subjetivo impacta positivamente a Intenção de Compra. Uma discussão associada ao tema bem-estar foi identificada no trabalho de Rana e Paul (20I7), visto que a expectativa de bem-estar associada às dimensões que a compõem motiva fortemente os consumidores a comprar alimentos orgânicos, sugerindo que o prazer e o bem-estar são os principais valores nas mentes dos consumidores. Entretanto, esse construto foi testado como expectativa pós-consumo e não como um antecedente como visto nesta pesquisa.

Analisando os resultados alcançados pelo modelo proposto nesta pesquisa com os de outros trabalhos citados a seguir e que incluíram novos construtos no modelo original da TCP, nota-se que a presente pesquisa de fato contribui na capacidade de explicação da variância da Intenção de Compra.

Além dessa capacidade de explicar a variância da intenção, o modelo de fato apresenta outros resultados que indicam respostas a questões como as levantadas por Scalco et al. (20I7). Por exemplo, Scalco et al. (2OI7) alegam que a força que rege a atitude sobre a intenção de compra ainda é incerta. Os resultados deste estudo mostram que o construto Bem-Estar Subjetivo responde essa lacuna da atitude, pois, considerando o contexto dos alimentos orgânicos e as pesquisas descritas até este ponto, indica a relevância desse construto e de suas dimensões. Desse modo, os resultados demonstram que, a partir do momento no qual o indivíduo reconhece os 
possíveis benefícios que o alimento orgânico lhe proporciona subjetivamente, há uma influência positiva que se correlaciona com as atitudes para que realmente esse sujeito crie uma expectativa sobre a necessidade desse tipo de alimento. Com isso, há um aumento da sua intenção em comprar e, consequentemente, efetiva-se o ato de aquisição.

\section{Conclusão}

O principal objetivo deste trabalho foi analisar se o construto Bem-Estar Subjetivo potencializa a intenção de compra de alimentos orgânicos. A escolha do tema foi realizada devido ao crescente consumo desse tipo de alimento e aos fatores que afetam essa relação de consumo, os quais vão além das questões apenas nutricionais que o alimento fornece. Desse modo, a fim de se cumprir o objetivo do trabalho, realizou-se uma pesquisa em diversas localidades, abrangendo um perfil mais amplo do consumidor de alimentos orgânicos, para que, assim, fosse possível coletar dados referentes à perspectiva do Bem-Estar Subjetivo, Atitudes, Normas Subjetivas e Controle Percebido desses consumidores. Identificou-se TCP e o Bem-Estar Subjetivo, possibilitando a conclusão de que o Bem-Estar Subjetivo possibilita o aumento da predição da intenção e age como um potencial antecedente frente principalmente na Atitude dos consumidores de alimentos orgânicos.

Os resultados indicam que o Bem-Estar Subjetivo é um preditor da Intenção de Compra de alimentos orgânicos e tem um papel relevante de influência frente aos construtos da TCP, indicando que o indivíduo considera a perspectiva do seu bemestar quando tem a intenção de comprar algum alimento de origem orgânica. Isso pois a ação de comprar o alimento orgânico envolve um complexo mecanismo de relações cognitivas, desde as crenças de saúde a processos emocionais, constituintes da sensação de bem-estar que cada indivíduo preza como relevante para a sua vida. Isto é, há consumidores que podem estar mais propensos a um Bem-Estar Subjetivo mais associado a preocupações físicas e nutricionais do alimento orgânico em tese, enquanto outros compram esse tipo de alimento levando em conta questões mais holísticas, como a preocupação com a sua consciência de preservação ambiental ou 
mesmo por um estilo de vida mais associado a elos emocionais e espirituais, que possibilitam a esses sujeitos se sentirem de bem com o seu próprio “eu”.

Desse modo, os resultados apresentados confirmam o aceite do modelo conceitual proposto, o qual atendeu aos objetivos específicos indicados. Assim, em relação ao perfil dos consumidores, notou-se que atualmente há um contexto de disseminação do consumo de alimentos orgânicos entre as classes sociais, mas há um perfil de consumidor que se estabeleceu ao longo do tempo, como foi descrito. Apesar de se observar que há mais indivíduos consumindo orgânicos, percebe-se que aqueles com maior renda e com níveis de gasto com a compra desse tipo de alimento são os que apresentaram as maiores médias de Bem-Estar Subjetivo. Isso indica que consumidores com maiores rendas e gasto com a compra apresentam uma sensação de Bem-Estar Subjetivo por meio do consumo de alimentos orgânicos maior do que aqueles que gastam menos com a compra desse tipo de alimento.

Quanto às contribuições teóricas, elas estão relacionadas aos estudos do BemEstar Subjetivo, com a aplicação de um instrumento direcionado para o contexto alimentar, aos estudos da TCP e à construção de um modelo conceitual, associando Bem-Estar Subjetivo, Atitudes, Normas Subjetivas e Controle Percebido frente à Intenção de Compra de alimentos orgânicos. O modelo proposto incorpora o construto do Bem-Estar Subjetivo como um dos preditores da Intenção de Compra, de modo que, na perspectiva acadêmica, esta tese contribui para a sequência de estudos que se direcionam a compreender as forças que impulsionam o modelo da TCP bem como a questão do papel do bem-estar do indivíduo relacionada ao contexto da sua alimentação (Scalco et al., 20I7; Apaolaza et al., 20I8; Ares et al., 20I6; Meiselmen, 20I6; Guillemin et al., 20I6). Além disso, essa pesquisa a sua relevância na linha de pesquisa sobre os motivos do consumo de alimentos orgânicos, visto que estudos abordando o termo Bem-Estar Subjetivo ainda são escassos e a inclusão do construto Bem-Estar Subjetivo em um modelo de predição que é amplamente utilizado, como é o caso da TCP, favorece a compreensão das forças que impulsiona as Atitudes, as Normas Subjetivas e o Controle Percebido. 


\section{Referèncias}

Ajzen, I. (I99I). The theory of planned behavior. Orgnizational Behavior and Human Decision Processes, 50(I), I79-2II.

Ajzen, I., \& Fishbein, M. (1977). Attitude-behavior relations: a theoretical analysis and review of empirical research. Psychological Bulletin, 84(5), 888-918.

Ajzen, I., \&Fishbein, M. (2005). The influence of attitudes on behavior, in Albarracín, D., Johnson, B. T., Zanna, M. P. (Eds.). The Handbook of Attitudes, Psychology Press, pp. I73-22I.

Al-Swidi, A., Huque, S. M. R., Hafee, M. H., \&Shariff, M. N. M. (2014). The role of subjective norms in theory of planned behavior in the context of organic food consumption. British Food Journal, II6(Iо), , 56

Apaolaza, V., Hartmann, P., D’Souza, C., \& López, C. M. (2018). Eat organic - Feel good? The relationship between organic food consumption, health concern and subjective wellbeing. Food Quality and Preference, $63(4), 55^{\mathrm{I}-62 .}$

Ares, G., De Saldamando, L., Giménez, A., Claret, A., Cunha, L. M., Guerrero, L., ..., \&Deliza, R. (20I5). Consumers' associations with wellbeing in a food-related context: A cross-cultural study. Food Quality and Preference, 40(I), 304-315.

Ares, G., Giménez, A., Vidal, L., Zhou, Y., Krystallis, A., Tsalis, G., ...\&Deliza, R. (20I6). Do we all perceive food-related wellbeing in the same way? Results from an exploratory cross-cultural study. Food Quality and Preference, $5^{2}(\mathrm{I}), 62-73$.

Arvola, A., Vassallo, M., Dean, M., Lampila, P., Saba, A., Lähteenmäki, L., \& Shepherd, R. (2008). Predicting intentions to purchase organic food: The role of affective and moral attitudes in the theory of planned behaviour. Appetite, $5 \mathrm{O}(4), 443-454$.

Chin, W. W. (1998). The partial least squares approach for structural equation modeling. In G. A. Marcoulides (Ed.), Modern methods for business research (pp. 295-236). Lawrence Erlbaum Associates.

Diener, E., Emmons, R. A., Larsen, R. J., \& Griffin, S. (1985). The satisfaction with life scale. Journal of Personality Assessment, 49(3), 7 T-75.

Diener, E., Scollon, C. N., \& Lucas, R. E. (2003). The evolving concept of subjective well-being. The multifaceted nature of happiness. Advances in Cell Aging and Gerontology, I5(2), I87-219.

Diener, E., Suh, E. M., Lucas, R. E., \& Smith, H. L. (1999). Subjective well-being: Three decades of progress. Psychological Bulletin, $125(2), 276-302$.

Festinger, L., \& Carlsmith, J. M. (1959). Cognitive consequences of forced compliance. Journal of Abnormal and Social Psychology, 58(2), 203-210.

Greenberg, J. S. (1985). Health and wellness: A conceptual differentiation. Journal of School Health, 55(Io), 403-406.

Guillemin, I., Marrel, A., Arnould, B., Capuron, L., Dupuy, A., Ginon, E. (20I6). How French subjects describe well-being from food and eating habits? Development, item reduction and scoring definition of the Well-Being related to Food Questionnaire (Well-BFQ@). Appetite, 96(Iо), 333-346. 
Hair Jr., J. F., Black, W. C, Babin, B. J., Anderson, R. E., \& Tatham, R. L. (2009). Análise multivariada de dados. (6a ed.). Bookman.

Hair Jr., J. F., Gabriel, M. L. D. D. S., \& Patel, V. K. (2OI4). Modelagem de Equações Estruturais Baseada em Covariância (CB-SEM) com o AMOS: Orientações sobre a sua aplicação como uma Ferramenta de Pesquisa de Marketing. Revista Brasileira de Marketing, I3(2), 44-55.

Krystallis, A., \&Chryssohoidis, G. (2005). Consumers' willingness to pay for organic food: Factors that affect it and variation per organic product type. British Food Journal, $\operatorname{IO} 7(5), 3^{20-343 .}$

Marôco, J. (2OIO). Análise de equações estruturais: fundamentos teóricos, software \& aplicações. Pero Pinheiro: Report Number.

Meiselman, H. L. (20I6). Quality of life, well-being and wellness: Measuring subjective health for foods and other products. Food Quality and Preference, 54(4), IOI-IO9.

Nuttavuthisit, K., \& Thøgersen, J. (2OI7). The Importance of Consumer Trust for the Emergence of a Market for Green Products: The Case of Organic Food. Journal of Business Ethics, I4O(5), 323-337.

Paul, J., Modi, A., \& Patel, J. (20I6). Predicting green product consumption using theory of planned behavior and reasoned action. Journal of Retailing and Consumer Services, 29(3), ı23-134.

Rana, J., \& Paul, J. (2OI7). Consumer behavior and purchase intention for organic food: A review and research agenda. Journal of Retailing and Consumer Services, 38(2), I57-I65.

Roscoe, L. J. (2009). Wellness: A review of theory and measurement for counselors. Journal of Counseling and Development, 87 (I), 216-226.

Scalco, A., Noventa, S., Sartori, R., \& Ceschi, A. (20I7). Predinting organic food consumption: A meta-analytic structural equation model based on the theory of planned behavior. Appetite, II2(I), 235-248.

Taufique, K. R., \& Vaithianathan, S. (2OI8). A fresh look at understanding green consumer behavior among young urban Indian consumers through the lens of Theory of Planned Behavior. Journal of Cleaner Production, I83(IO), 46-55.

Teng, C-C., \& Wang, Y-M. (20I5). Decisional factors driving organic food consumption: Generation of consumer purchase intentions. British FoodJournal, II7 (3), IO66-IO8I.

Yadav, R., \& Pathak, G. S. (20I6). Young Consumers' Intention Towards Buying Green Products in a Developing Nation: Extending the Theory of Planned Behavior. Journal of Cleaner Production, I35(I), 732-739. 